Article

\title{
A Solution Based on Bluetooth Low Energy for Smart Home Energy Management
}

\author{
Mario Collotta * and Giovanni Pau \\ Faculty of Engineering and Architecture, Kore University of Enna, Cittadella Universitaria, \\ Enna 94100, Italy; E-Mail: giovanni.pau@unikore.it \\ * Author to whom correspondence should be addressed; E-Mail: mario.collotta@ unikore.it; \\ Tel.: +39-0935-536494; Fax: +39-0935-536623.
}

Academic Editor: Nyuk Wong

Received: 28 August 2015 / Accepted: 3 October 2015 / Published: 21 October 2015

\begin{abstract}
The research and the implementation of home automation are getting more popular because the Internet of Things holds promise for making homes smarter through wireless technologies. The installation of systems based on wireless networks can play a key role also in the extension of the smart grid towards smart homes, that can be deemed as one of the most important components of smart grids. This paper proposes a fuzzy-based solution for smart energy management in a home automation wireless network. The approach, by using Bluetooth Low Energy (BLE), introduces a Fuzzy Logic Controller (FLC) in order to improve a Home Energy Management (HEM) scheme, addressing the power load of standby appliances and their loads in different hours of the day. Since the consumer is involved in the choice of switching on/off of home appliances, the approach introduced in this work proposes a fuzzy-based solution in order to manage the consumer feedbacks. Simulation results show that the proposed solution is efficient in terms of reducing peak load demand, electricity consumption charges with an increase comfort level of consumers. The performance of the proposed BLE-based wireless network scenario are validated in terms of packet delivery ratio, delay, and jitter and are compared to IEEE 802.15.4 technology.
\end{abstract}

Keywords: smart home automation; home energy management; fuzzy logic controller; wireless networks; bluetooth low energy 


\section{Introduction}

In recent years, with the introduction of the Internet of Things (IoT), the research and the implementation of home automation are getting more popular [1], because the IoT holds promise for making homes smarter. A smart home is a residential dwelling equipped with sensors and possibly actuators to collect data and send controls according to occupants' activities and expectations. Home automation (HA) refers to the mechanization and automatic control of various residential activities. Typically, HA provides a centralized control of electrical appliances, such as air conditioners, lighting and security systems and even a home theater. Adding intelligence to the home environment, it would be possible to obtain excellent levels of comfort, and another feature taken into account is the energy savings.

It is necessary to highlight that an important role is also played by consumers, because, as shown in [2], the reduction of energy consumption can be obtained by only providing the consumption profile of appliances to the consumers and accordingly helping them to change their behavior. This approach, known as demand-side management (DSM) [3], based on bi-directional communication is enabled by the smart grid in order to smooth the load curve of the traditional grid. As a consequence, DSM programs influence the behavior of the consumers regarding electricity consumption because the DSM technique mainly relies on matching present generation values with demand by controlling the energy consumption of appliances and optimizing their operation at the user side. For instance, a practical usage can be the shifting of "wet appliances" from peak time to off-peak time. In literature are shown other approaches based on Time-of-Use (TOU) [4] pricing. The consumers, knowing a priori the energy price, could chose the best time to switch on or off the home appliances in order to improve (reducing) the energy cost.

However, the integration of several electrical devices in the household is an open challenge because of the absence of an inexpensive and standardized communication protocol between them [5], even thanks to the introduction and the use of renewable energy [6]. Wireless Networks (WNs) are getting more integrated to our daily lives, recently in smart homes WNs are using to increase the inhabitant comfort [7] and they can receive the user feedbacks in order to meet the itself comfort. Several wireless technologies, such as Bluetooth Low Energy (BLE) [8], IEEE 802.15.4/ZigBee [9] and IEEE 802.11/Wi-Fi [10], have been proposed in order to embed various levels of intelligence for smart homes. In fact, in this research field, several literature works have been presented. For instance, the authors of [11] introduce a prototype that allows users to visually define the behavior of a $\mathrm{WN}$ in a smart home. The prototype includes relevant system features, such as an IoT-enabled sensor and actuator network, a user-friendly programming interface, a support for device and resource discovery and over-the-air code generation and deployment. A management system based on a WN for home automation and wellness is proposed by [12]. This system allows the consumers to control and to manage both the daily activity of home appliances and the home climate characteristics with a consequent reduction of energy consumption. The proposed system has good modularity and configurability characteristics with very low power consumption. The authors of [13] propose a fuzzy logic approach utilizing a wireless network and smart grid incentives in order to reduce the residential load demand. Simulation results show that the their proposed approach reduced the residential load demand without jeopardizing occupants' thermal comfort based on the information received from sensors, the grid and the occupants' preferences. Considering 
that the current energy grid is evolving into a smart grid, where consumers can directly reach and control their consumption, WNs can take part in domestic energy management systems, as well.

The use of power line communication (PLC) can be another way to let the home energy management (HEM) system communicate with home appliances and control them [14]. Several literature works have been published in the last few years. For instance, the smart home control network proposed in [15] employs the PLC as the network backbone and the WN for data sensing. A prototype of the proposed smart home control network with smart lighting control is implemented, while simulations and practical experiments show that the implemented prototype system works well and that the proposed smart home control network provides a good packet failure rate and considerable energy savings. Another home energy management system is introduced in [16] and considers also the energy generation. In this case, PLC modems are installed in each solar panel to monitor its status. Therefore, the main aim of PLC technology is the monitoring of each solar panel for maintenance. On the contrary, an accurate analysis of a PLC application in a smart home is presented in [17]. The authors present a new method in order to estimate the impedance of the home power line, in the Federal Communications Commission band, and then to solve some problems in this area.

However, some issues need to be analyzed. The two key issues associated with PLC are firstly one relating to the cabling configuration and the second to interference. In order for the power line to work, the connecting devices need to be physically located on the same circuit. However, this is not always the case, as many houses have separate circuits for the three different phases of electric power that comes to the home to reduce the load on each wire. For instance, such a design is particularly common in new buildings where the need for increased power necessitates the use of multiple circuits. In these circumstances, power line technology will not work throughout the whole home. It is known that wireless networks do not have major problems in device positioning, precisely thanks to their "wireless" nature. Regarding radio interference and fading problems, it is necessary to note that these issues can be found also in PLC, due to the leakage of RF signals. As the number of power line devices in the same area increases and power line technology is being used by multiple households, the signals are being shared among multiple users, and the risk of interference and degradation occurs, leading to a reduction in the throughput of data, as the signals are all sharing the same frequency. Other electrical equipment also injects noise into the electrical wires, resulting in degradation in the performance of nearby PLC networks. Wireless networks are useful for gathering environmental data, and they can be also a valid solution for controlling home appliances. A wireless network is not constrained by the existence nor quality of the existing cabling infrastructure. It offers good speeds, greater predictability and quality and the option of moving devices at will.

To cope with HEM, in this paper, a novel solution is introduced. In fact, it is clear that an HEM implementation can lead to a socially- and economically-beneficial environment by addressing consumers' and utilities' concerns. For instance, increased savings, better peak load management and the reduction in peak-to-average ratio are some of the benefits that can be achieved by the solution proposed in this paper. This proposed solution is based on the communication among home appliances, a central energy management unit (EMU), a smart meter and a storage unit inside a smart home. Furthermore, the communication among these entities is based on a WN using the Bluetooth Low Energy (BLE) protocol [8]. In fact, the choice of a wireless protocol with respect to another one may involve significant 
benefits and quite different results. The novelty introduced in this paper is both the use of an HEM scheme and mainly the use of BLE in the wireless network, which coordinates the communication between home appliances. The benefits of the proposed solution are confirmed by validations, whereas, to the best of our knowledge, there are no works in the literature in which BLE is applied in a wireless network that supports a fuzzy-based HEM scheme.

The paper is organized as follows. In Section 2, wireless protocols commonly used for home automation are analyzed in order to explain why BLE has been chosen in the solution proposed in this paper. In Section 3, the proposed solution is introduced, presenting the wireless network architecture (Section 3.1), the fuzzy-based algorithm for HEM (Section 3.2) and the fuzzy logic controller (Section 3.3). In Section 4, the performance obtained by the proposed solution is shown, and finally, in Section 5, the paper is summarized, reporting conclusions and future works.

\section{BLE vs. Other Wireless Solutions}

Before introducing the solution proposed in this paper, it is necessary to carry out an analysis of the wireless protocols commonly used in home automation. In fact, the choice of the wireless protocol is critical, because it determines the performance of the entire network on which the HEM solution is based. Some of the important features when selecting the appropriate short-range wireless technology for smart homes are the following:

- cost of the radio technology: as many of the devices are small low-cost devices, the radio must not add too much additional cost to the bill-of-material; this also implies that the radio and device application in many cases need to share the same computing engine (micro-controller).

- power consumption: many use cases require a battery or some kind of energy harvesting technology as a power source.

- ease-of-use: it must be easy to associate a device with the network and with the Internet services.

- security: the authentication and the encryption must be adequately supported by the wireless technology, and sometimes, end-to-end. security (all the way from sensors to the web services) is required.

- available ecosystem: the possibility to connect to smart phones, tablets, PCs, home gateways, etc., is important; this requirement also drives volumes and has an important impact on the cost (a good example is Bluetooth Classic, where the large volumes of phones and phone accessories have lowered the costs).

- range: it is necessary to have the capability to cover enough of a range or to have some capabilities to extend the coverage (repeaters, routers, etc.) without having too big of an impact on the system cost.

Table 1 shows how different wireless technologies fit specific verticals. It is useful to note that the green check-mark indicates that the protocol behaves well in the reference application, unlike the orange color, which indicates just a satisfactory behavior. On the contrary, the red cross indicates that the protocol is not suitable for that application. According to Table 1, the following conclusions can be deduced: 
- all three technologies have built-in link layer authentication and encryption, which sometimes needs to be completed with end-to-end security from the sensor to the web application;

- correctly used, Bluetooth Low Energy has the potential for less power consumption than IEEE 802.15.4 (less overhead);

- the lack of native support for IEEE 802.15.4 in mobile devices (smart phones, tablets, laptops, etc.) is a problem, especially for mobile or temporarily mobile use cases;

- one of the major advantages of the technology for the smart grid area is the existing Bluetooth ecosystem, which makes it seamless for consumers to start using BLE in their smart grid devices. Bluetooth is already in mobile phones and many personal computers, which can provide the necessary user interface for smart energy applications. Thanks to Bluetooth, the integration of a smart ecosystem throughout the home could be made very easy and very fast;

- BLE and IEEE 802.11 devices are easy to associate with a network and mainly with Internet services, while this is often more difficult with IEEE 802.15.4 devices;

- IEEE 802.15.4 has a main advantage in its range, since many IEEE 802.15.4-based technologies (e.g., ZigBee) support meshing, whereby the coverage can be extended by using routers;

- reliable delivery of information is critical in smart home networks to obtain accurate automation functionality. Error detection and correction enable the identification and correction of the corrupted data in unpredictable communication channels. IEEE 802.15.4, IEEE 802.11 and Bluetooth use cyclic redundancy check (CRC) to validate the packets. In fact, a certain number of bits (checksum) is appended to the message being transmitted. Different technologies use 16, 8, 32 checksums, and the higher the checksum, the more powerful the error controls. IEEE 802.11 shows the highest CRC error control proficiencies by offering 32 checksums, while IEEE802.15.4 and Bluetooth use 16 checksums. To find out that the data were not infected in transmission, the receiver determines whether the check bits agree with the data with a certain degree of probability. If the CRC shows that the packet was not corrupted, ACK will be sent or, otherwise, the packet is dropped and retransmission needed. All three protocols have good reliability;

- BLE is very reliable with its support for adaptive frequency hopping (AFH) and other features inherited from Bluetooth Classic;

- IEEE 802.11/Wi-Fi can be used in devices with less demands on low power consumption and as a wireless backbone in combination with other technologies.

- as shown in [5], the transmission rates of all three protocols are suitable for all common applications in a smart home; it is useful to remark that the transmission rate of the considered protocols are $1 \mathrm{Mbps}$ for BLE, $250 \mathrm{kbps}$ (2.4 GHz) for IEEE 802.15.4 and 11-65-450 (IEEE $802.11 \mathrm{n})$ Mbps for IEEE 802.11.

BLE has a high potential in becoming an important technology for smart homes in low power, low cost and small devices. However, there are still use cases where IEEE 802.15.4-based technologies are used especially in areas where it is already established. In spite of its installed base in smart energy, home and building automation applications, IEEE 802.15.4 faces competition in BLE in these applications, as well. Anyhow, BLE has a lower energy consumption than IEEE 802.15.4 and, for this reason, may be the best choice in applications of home automation. IEEE 802.11 is used in devices where cost and low power are less important and as a wireless backbone combined with the other wireless technologies. In 
the end, through the analysis carried out in this section, it is clear why in this paper BLE has been chosen for the proposed HEM solution.

Regarding the integration of BLE in home appliances, it is possible to chose different solutions. In fact, the easiest way is to use smart plugs, the cost of which can range from $30 €$ to $100 €$, whether or not they are programmable. Another solution is to use COTS (commercial off-the-shelf) hardware that has to be programmed by a "geek" user. In this case, the use difficulty may be higher, because the user has to develop his BLE network from scratch. However, on the other hand, besides having possibly greater ultimate satisfaction, it is possible to obtain also lower realization costs. In fact, in order to create a prototyping board (micro-controller, radio transceiver, etc.), based on BLE, with the aim to interact with the home appliances, the development cost is around $20 €$. Anyhow, it is also possible to use several ready solutions on the market, such as [18-22], whose cost can range from $100 €$ to $500 €$.

Table 1. Comparison of wireless technologies and their usefulness in smart homes. BLE, Bluetooth Low Energy.

\begin{tabular}{cccc}
\hline & BLE & IEEE 802.15.4 & IEEE 802.11 \\
\hline Cost & $\checkmark$ & $\checkmark$ & $\checkmark$ \\
Security & $\checkmark$ & $\checkmark$ & $\checkmark$ \\
Power consumption & $\checkmark$ & $\checkmark$ & $x$ \\
Ecosystem & $\checkmark$ & $x$ & $\checkmark$ \\
Reliability & $\checkmark$ & $\checkmark$ & $\checkmark$ \\
Ease of use & $\checkmark$ & $\checkmark$ & $\checkmark$ \\
Range & $\checkmark$ & $\checkmark$ & $\checkmark$ \\
Transmission rate & $\checkmark$ & $\checkmark$ & $\checkmark$ \\
\hline
\end{tabular}

\section{The Proposed Solution}

In the literature, several approaches based on WNs for smart grids and smart homes have been proposed $[23,24]$. In fact, advanced information and communications technologies (ICTs), monitoring and control, energy harvesting [25] and innovative metering technologies via smart wireless devices are becoming increasingly important. For instance, in [26], a domestic energy management scheme based on a wireless sensor home area network (WSHAN) is introduced. This scheme focuses on the communication among smart appliances and an EMU through the wireless network, and consumer demands are processed in near real time. On the contrary, the approaches proposed in [27,28] aim at a TOU pricing benefit in order to decrease the energy cost. The goal of these authors is to shift the consumer load to off-peak periods using the WN to deliver their requests to the EMU. However, although the analyzed WN-based works (from [23] to [28]) obtain good performance, their authors do not bother to choose the most appropriate wireless protocol. In fact, as mentioned above, the choice of a proper wireless protocol with respect to another one may involve significant benefits and quite different results.

The system proposed in this paper is depicted in Figure 1 and consists of various energy sources, storage devices, electrical appliances, a control unit and related communication tools. As it is possible 
to see, the smart home is able to generate its own energy thanks to renewable energy sources, such as wind turbine and solar photovoltaic panels, and stores this energy by means of batteries. It is necessary to highlight that the renewable power forecasting methodology is not addressed in this paper, because it is not the main aim of this work. However, it is useful to note an important feature in the considered smart home. In fact, in the interaction between the EMU and the storage devices, a key role is played by a "charging station", a kind of inverter, that converts direct current electricity from renewable energy sources or a home battery into the alternating current used by home's lights, appliances and devices. Anyhow, it is obvious that even the energy from a high voltage power line can be used. However, it is clear that in homes, the high voltage is not used. For this reason, transformer stations, outside of the housing, are used for the transformation from high to low voltage.

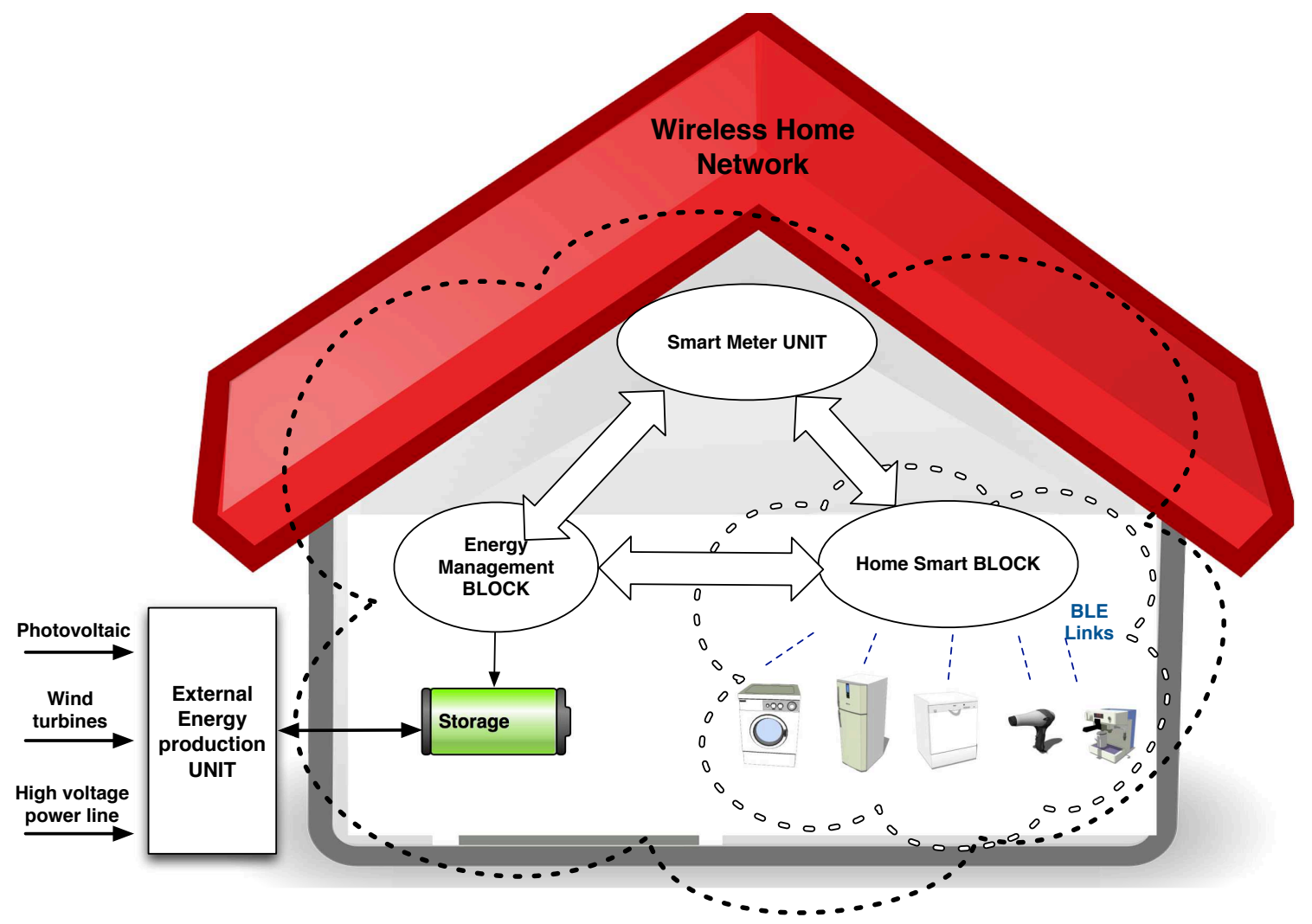

Figure 1. The proposed energy management system for smart homes.

The communication-based domestic energy management solution proposed in this work aims at reducing home electricity consumption charges, decreasing the electricity bill of the consumer by shifting the appliances' operation from peak demand hours to off-peak ones. In fact, the electrical appliances that are part of the home system can be used at various time periods every day depending mainly on the day of the week. In fact, there are substantial differences in the usage time of appliances throughout the day, especially between working days and the weekend. For instance, a refrigerator can be active for a $25 \mathrm{~min}$ period every 50 min because of its operation principle, and similarly, an air conditioner can be operated for 15 min every half an hour only in the summer months.

The system introduced in this paper, shown in Figure 1, involves communications among smart appliances, a central EMU and a WN. As it is possible to see, the decision making center is represented by 
the EMU, where a fuzzy-based algorithm, described in Section 3.2, deals with the HEM. The consumer may turn on any appliance at any moment of the day without taking into account that, at that moment, it can be also in peak hours. The proposed fuzzy-based algorithm allows the switching on of the appliance or suggests to the consumer which is the more appropriate start time. In fact, when an appliance is turned on, it sends a request to the EMU that checks the available stored energy in the storage system. Moreover, the EMU communicates also with the smart meter in order to know about the updated prices in that time slot. When the EMU receives the information about the amount of stored energy, it schedules a convenient start time for the appliance according to the proposed fuzzy-based algorithm and notifies the consumer. At this stage, the consumer can decide whether to accept the schedule proposed by the EMU, for instance the time period falls during peak hours or the level of stored energy is not enough, or to ignore it, switching on the appliance.

The proposed approach is not limited to the appliances that operate mostly autonomously and on a periodic basis, such as refrigerators, washers, etc. In fact, the proposed approach is valid and enforceable on all household appliances. For instance, considering a specific case, the system could suggest, through the smart plug, to wait after the consumer is about to start drying his or her hair after taking a shower when connecting and turning on the hair dryer, whereby the consumer can reject any scheduling proposal since he or she has the need to use the hair dryer at that precise moment. On the contrary, the consumer could prefer to remain with wet hair (or dry it with a towel) in order to shift the appliance cycle to hours where electricity prices are comparatively low. A different conclusion can be deducted if the appliance taken into account is not a hair dryer, but a vacuum cleaner, an iron, etc., for which, for example, if the consumer agrees, it is possible to postpone its use following the timing suggested by the proposed algorithm. This run-time mechanism of acceptance or rejection of the schedule can be managed through smart plugs. In this paper, smart plugs have not been analyzed in detail, because the main aim is to present the entire energy management approach. In any case, it is obvious that on the market, there are several devices that allow one to implement this mechanism of interaction between the consumer and household appliances that is introduced in this paper.

\subsection{Wireless Network Architecture}

Bluetooth Low Energy [8] operates in the $2.4 \mathrm{GHz}$ ISM band with only 40 channels spaced $2 \mathrm{MHz}$ apart. It is capable of transmitting at a rate of $1 \mathrm{Mbit} / \mathrm{s}$ using GFSK modulation. Like Bluetooth Classic, it uses frequency hopping, but it uses an adaptive frequency hopping and has a slower rate. BLE uses three of the 40 channels to advertise, which allows the device discovery. After a device is discovered and connected, the remaining 37 channels are used to transmit data. In a piconet of Bluetooth Classic, each master device establishes the frequency hopping sequence and can have up to seven slave connections. There is no such limitation in BLE, and in fact, "theoretically", to a master node can be connected "hundreds" of slaves. The relatively short transmission distances between the electrical appliances and the limited amount of data to be transmitted in a certain time interval can be deemed as other important factors in the selection process of the BLE protocol. It is useful to note that the range of BLE radio may be optimized according to the application. The majority of Bluetooth devices on the market today include the basic 30-foot (or 10-meter) range of the Bluetooth Classic radio, but there is no limit imposed 
by the specification. With BLE, manufacturers may choose to optimize the range to 200 feet (about 67 meters) and beyond, particularly in home sensor applications, where a longer range is a necessity.

The proposed network architecture, shown in Figure 2, is composed of several independent wireless cells (WCs), managed by a BLE master device that acts also as the EMU and integrates the smart meter module. The whole WN is composed of all of the WCs within which are the field devices (FDs), which are Bluetooth Low Energy devices dealing with a specific task, or are associated with home appliances through smart plugs. It is easy to deduce that in the proposed network architecture, some of the WCs behave as wireless sensor networks (WSNs), because they can continuously monitor environments with less human effort and are low cost and low power. In fact, through wireless communication, the master node receives and processes data detected by FDs placed inside the WC. It also allows sending user commands or system commands to FD nodes. Furthermore, wireless links allow the communication among mobile devices (such as smart phones or tablets) and the WCs. As a consequence, people can authenticate themselves inside the home automation system and subsequently monitor data detected by sensor nodes and, if needed, send commands.

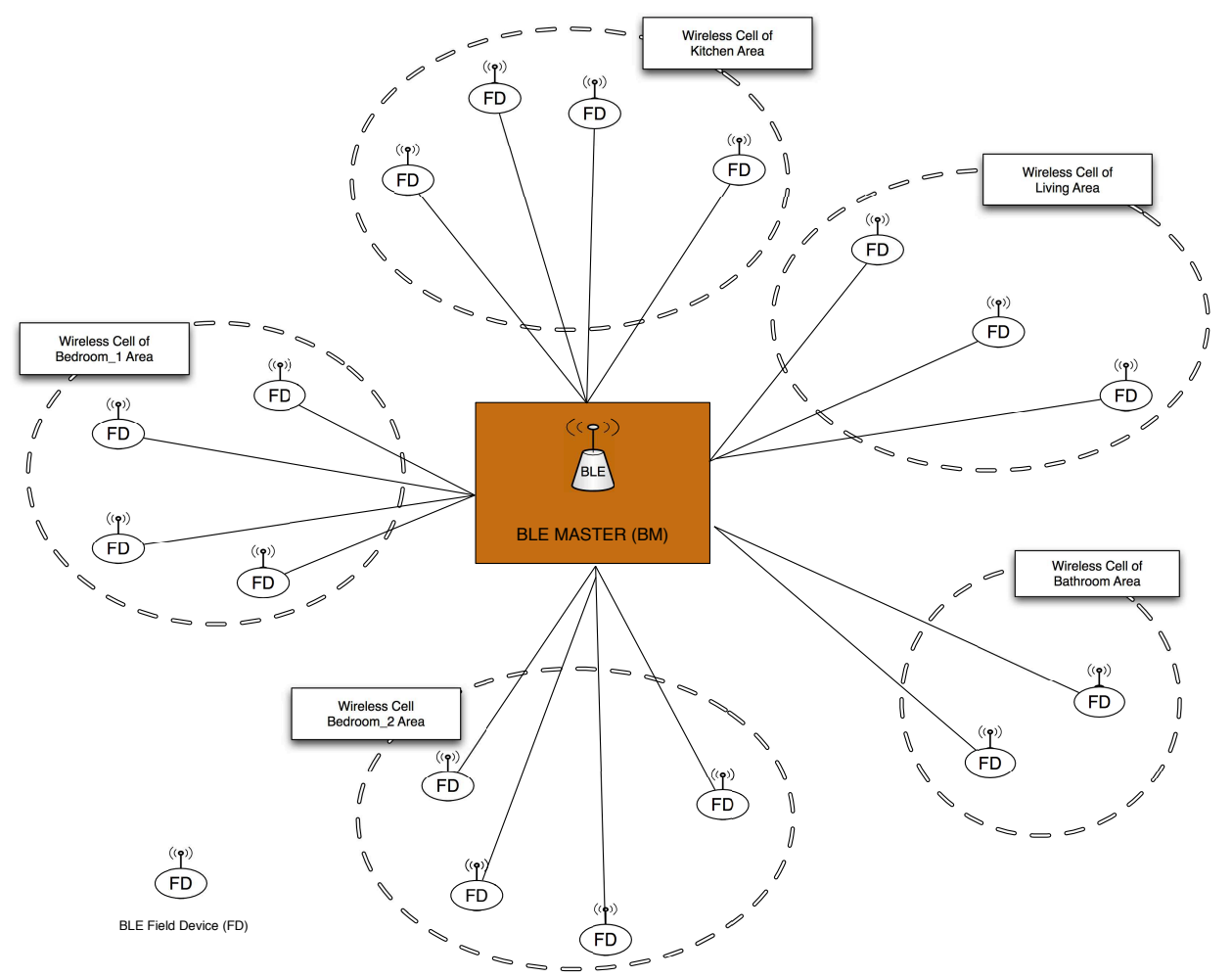

Figure 2. The proposed BLE-based wireless network topology.

Considering Figure 2, it is useful to evaluate the blocking effects between BLE master and slaves, due to walls or obstacles in rooms. Thanks to a modified modulation, BLE has an approximately $3 \mathrm{~dB}$ better link budget compared to Classic Bluetooth. A BLE unit can thereby offer a range of $200-300 \mathrm{~m}$ in the line of site without the need for an additional power amplifier. It is important in the aspects of robustness and reliability to have an appropriate placement of several devices in order to bridge temporary obstacles and interference. However, home sensors and actuators often need a range of 20-50 m, whereby it would be not necessary to use power amplifiers. On the contrary, considering that the range of BLE 
radio may be optimized according to the application, BLE manufacturers may choose to optimize the range, because there is no limit imposed by the BLE specification.

The proposed WN enables a variety of use cases; for this reason, a non-exhaustive list of examples is provided below:

- smart energy: window shades, HVAC, central heating, and so on, may be controlled depending on the information collected by several types of sensors that monitor parameters, such as temperature, humidity, light and presence; as a consequence, unnecessary waste of energy can thus be avoided.

- smart meters: they can be used to detect usage peaks and alert the household devices that may be causing them.

- light control: lights can also be activated in response to a command from remote control; furthermore, they can be turned on automatically when the presence and luminance sensors detect that people are in a poorly-illuminated room.

- safety: several sensors e.g., smoke detectors, glass-break sensors, and motion sensors can be installed in order to detect possible risk situations that trigger appropriate actions in response.

- remote control: infrared technology has been used for wireless communication between a remote control and devices such as TVs, Hi-Fi equipment, and heating, ventilating, and air conditioning systems; however, it requires both line-of-sight and short-distance communication. Therefore a radio frequency technology based on BLE can overcome these limitations, and it can be integrated in the home network architecture.

\subsection{Fuzzy-Based Algorithm for Home Energy Management}

The proposed fuzzy-based algorithm monitors and controls the electrical appliances in the smart home, planning a convenient start time for them. In order to realize a cost-effective operation, the management algorithm deals with guaranteeing the supply-demand balance by taking into account the present electricity price and the state of energy storage system. The flowchart of the proposed HEM algorithm is shown in Figure 3.

The starting point is represented by the time instant in which the consumer turns on an appliance $\left(T_{\text {start }}\right)$. At this time, a proper signal is sent to the EMU in order to notify about this operation. Subsequently, the EMU interacts both with the smart meter and with the storage system in order to know about the TOU prices. As a consequence, the EMU is able to known the corresponding energy consumption prices at that particular moment and then can easily check whether the current time falls within peak hours.

In the next step, the EMU verifies if the starting time of the appliance $\left(T_{\text {start }}\right)$ falls outside the peak hours. If so, the EMU allows the appliance to start immediately; otherwise, the algorithm moves to the next step, because $T_{\text {start }}$ is in peak hours. Considering that even standby appliances play a reasonable role in energy wastage [29], in the next step, the EMU checks for all of the standby appliances in home and turns them all off, regardless of their requests to be turned on.

Subsequently, the EMU communicates with the local energy storage system in order to inquire about the locally-generated or stored energy. As a consequence, if there is enough energy in the storage system for the appliance, it is started immediately, without any delay. On the contrary, the algorithm goes ahead 
to the next step where a comparison between the power ratings is carried out. In fact, considering that in the proposed scheme, a threshold value of power $\left(P_{t h r}\right)$ is set, the EMU evaluates the power rating of the i-th appliance $\left(P_{a p p}\right)$, and for every appliance request, its power rating is compared to the threshold value $\left(P_{t h r}\right)$. If $P_{a p p} \leq P_{t h r}$, then the appliance can start immediately; otherwise, the algorithm moves to the next step.

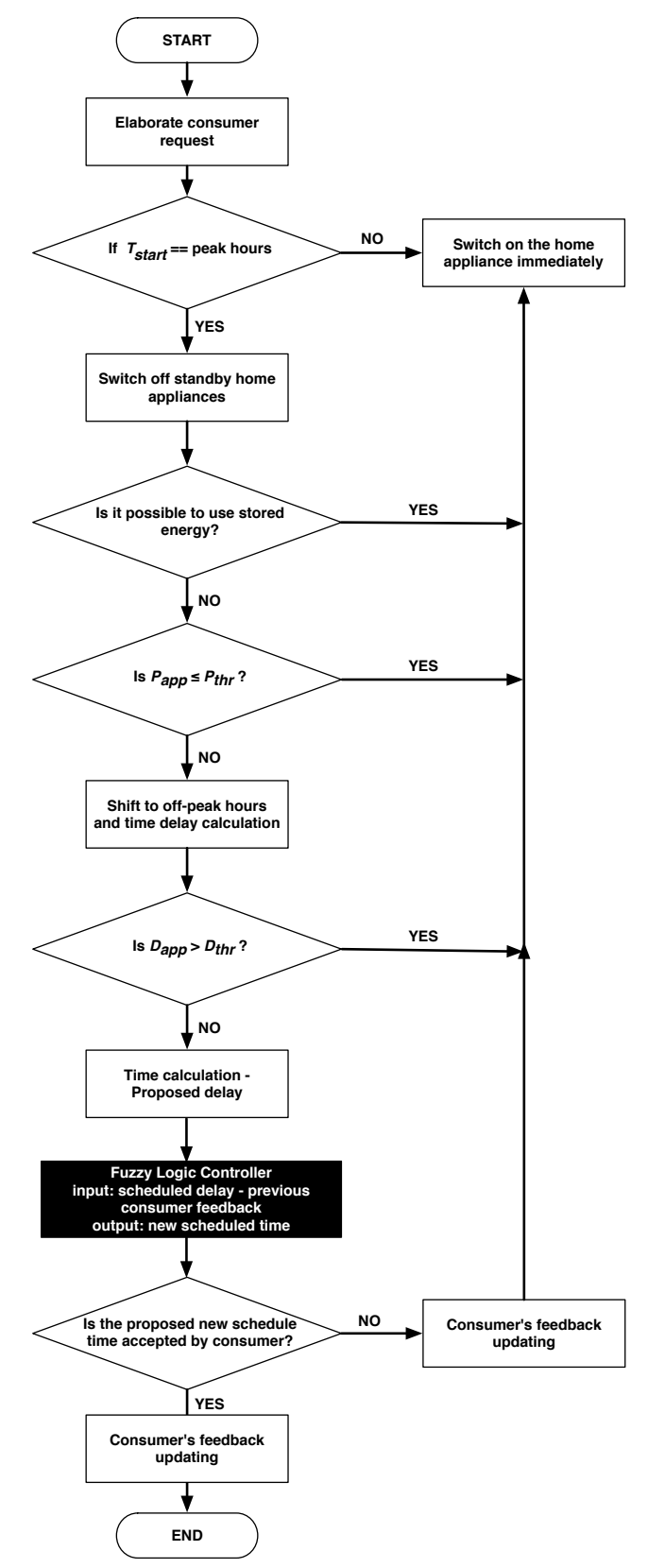

Figure 3. Home energy management (HEM) algorithm flowchart.

In this case, the appliance operation is shifted from peak hours to off-peak ones. As a result, a delay $\left(D_{a p p}\right)$, equal to the difference of the scheduled time suggested by the EMU and the request start time, is introduced in the operation of the appliance cycle due to the load shifting technique. It is necessary to note that this delay can be inversely proportional to the comfort level desired by the consumers. In fact, large delays can significantly lower their satisfaction. For this reason, in the proposed scheme, a threshold value of delay $\left(D_{t h r}\right)$ has been taken into account. Each device has its threshold value of delay. 
In fact, in the next step of the algorithm, a comparison between the delays is carried out. If $D_{a p p}>D_{t h r}$, then the appliance can start immediately.

The algorithm moves ahead in the next step if the previous condition is not satisfied. As a consequence, the appliance cycle is shifted to hours where electricity prices are comparatively low. Then, the EMU calculates the delay in the appliance cycle and sends it to the appliance in order to make it known what will be its start time. The scheduled time (delay) is calculated from the difference between the starting time of the appliance scheduled by the EMU and the request start time by the appliance. Subsequently, the scheduled time and the feedback previously provided by the consumer represent the input values of an FLC, described in detail in Section 3.3, that outputs the updated value of the scheduled time. Then, this value is both returned to the appliance, and the customer is notified.

In the next step, the consumer may deny or accept the schedule proposed by the EMU. In fact, the consumer may have the need to use the appliance at that time and therefore rejects the schedule given by the EMU, which possibly introduces a certain delay, and starts the appliance although it is in peak hours. In both cases, the EMU notifies the storage system and the smart meter about the consumer decision. It is obvious that the scheduling accepted by the consumer introduces a delay that is surely lower than the maximum threshold of the delay of the device.

\subsection{Fuzzy Logic Controller}

Fuzzy logic controllers (FLCs) [30] are becoming increasingly popular in everyday life. Home automation, health, industry and intelligent transportation systems (ITS) are some examples of application fields. In fact, FLCs can be appropriate for various engineering problems, especially for several problems where classical control methods do not achieve comparatively favorable results.

A general representation of the FLC introduced in this work is depicted in Figure 4. Although the representation is simple and general, it shows clearly what are the input parameters of the FCL and also the one that returns as output. The proposed system involves small data exchanges, because the data to be transferred to the controller are the calculated scheduled delay and the value of the consumer feedback related to the considered appliance. These inputs are used to update the value of the scheduled delay, returned as the output of the FLC, taking into account the feedback provided by the consumer. This fact entails a lower computational cost than, for instance, other approaches based on more complex fuzzy controllers (such as Type-2 fuzzy, Petri net, etc.), other approaches that use different controllers in parallel (for instance, a controller in each cluster in the network) or other approaches that consider several parameters as input to the controller. These properties of the proposed FLC not only allow one to implement the solution introduced in this paper on low cost hardware, but are also expected to be beneficial in terms of lower data processing times and lower control actuation times for the fuzzy logic controllers.

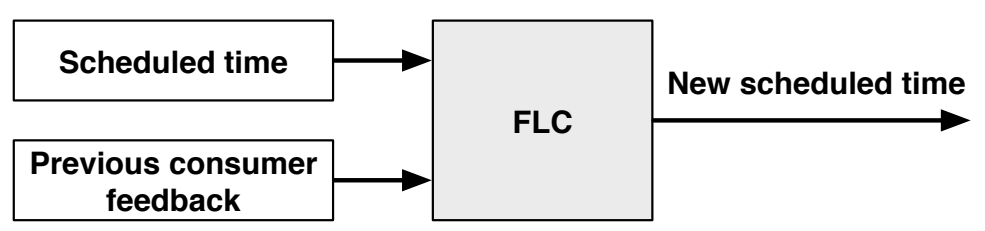

Figure 4. Proposed fuzzy logic controller (FLC) architecture. 
A FLC is based on membership functions (MFs), which are curves that define how each point in the input space is mapped to a membership value (or degree of membership) between zero and one. The input space is sometimes referred to as the universe of discourse. An MF can have different shapes; however, in this paper, triangular MFs have been considered. Considering a generic variable $x$ containing a range of values from the minimum to the maximum value that the variable itself can assume, each membership function can be represented by the generic triangular-shaped membership function [31] as follows:

$$
\mu_{A}(x)= \begin{cases}0 & \text { if } x \leq a \\ \frac{x-a}{m-a} & \text { if } a<x \leq m \\ \frac{b-x}{b-m} & \text { if } m<x<b \\ 0 & \text { if } x \geq b\end{cases}
$$

where $a$ is a lower limit; $b$ is an upper limit and $m$ is a value, with $a<m<b$. A generic triangular-shaped MF is depicted in Figure 5.

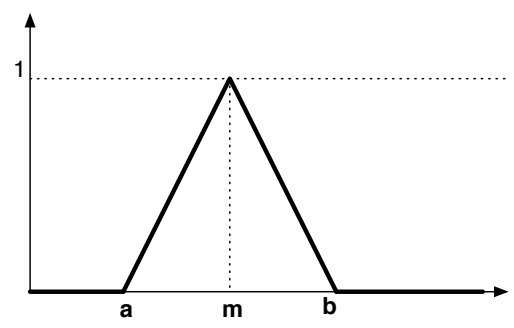

Figure 5. Generic triangular-shaped membership function.

The proposed FLC considers three membership functions (Low, Medium, High) for the scheduled time, both for the input variable and for the output one. These functions fuzzify the crisp inputs, while the ranges of which depend on the appliance type. In fact, each appliance has different values of maximum allowable delay. For instance, considering a generic appliance with a maximum allowable delay of $10 \mathrm{~h}$, the triangular membership functions of the scheduled time are depicted in Figure 6, where the degree of membership is represented by normalized values $[0,1]$.

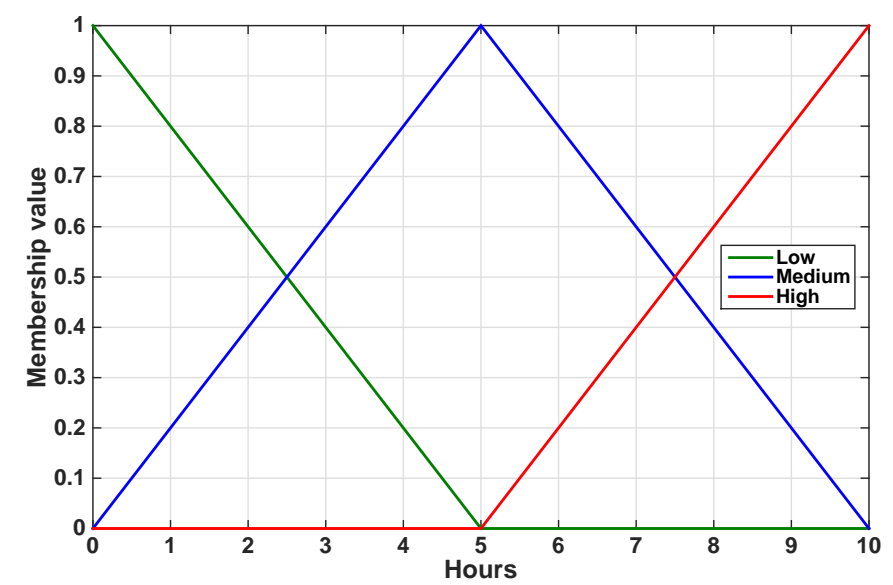

Figure 6. Triangular membership functions for the scheduled time of the input and output parameters for the FLC. 
Regarding the feedback provided by the consumer, it is not possible to obtain a numerical value. In fact, the consumer can accept or deny the schedule proposed by the algorithm. For this reason, in order to determine the value of the consumer feedback to use as the input parameter of the FLC, the inference rules shown in Table 2 are used. For instance, considering the rule 3, if the consumer decision is Accept and the scheduled delay is High, then the feedback value will be High. The feedback value is updated each time the consumer makes a decision and will be used by the algorithm and by the FLC later when the same appliance will be re-used.

Table 2. Inference rules for consumer feedback.

\begin{tabular}{cccc}
\hline Rule & Consumer decision & Scheduled time & Feedback value \\
\hline 1 & Accept & Low & Low \\
2 & Accept & Medium & Medium \\
3 & Accept & High & High \\
4 & Deny & Low & Medium \\
5 & Deny & Medium & High \\
6 & Deny & High & High \\
\hline
\end{tabular}

Finally, as shown in Table 3, the output value is determined through nine fuzzy rules based on the IF-THEN statement of classic programming languages. For instance, considering the rule 7 , if the scheduled time is High and the consumer feedback is Low, then the new scheduled time will be Medium. The outputs of the inference mechanism are fuzzy output variables. The FLC must convert its internal fuzzy output variables into crisp values, through the defuzzification process, so that the actual system can use these variables. Defuzzification can be performed in several ways. In this paper, the centroid of area (COA) method [32] has been chosen. In this method, the centroid of each membership function for each rule is first evaluated. The final output is then calculated as the average of the individual centroid weighted by the membership values as follows:

$$
\text { new_scheduled_time }=\frac{\sum_{i=1}^{n} O u t_{i} * C_{i}}{\sum_{i=1}^{n} C_{i}}
$$

where $O u t_{i}$ is the output of rule base $i$ and $C_{i}$ is the center of the output membership function. 
Table 3. Inference rules for the new scheduled delay.

\begin{tabular}{cccc}
\hline Rule & $\begin{array}{c}\text { Antecedent } \\
\text { scheduled time }\end{array}$ & $\begin{array}{c}\text { Antecedent } \\
\text { consumer feedback }\end{array}$ & $\begin{array}{c}\text { Consequent } \\
\text { scheduled time }\end{array}$ \\
\hline 1 & Low & Low & Low \\
2 & Low & Medium & Low \\
3 & Low & High & Medium \\
4 & Medium & Low & Medium \\
5 & Medium & Medium & Medium \\
6 & Medium & High & High \\
7 & High & Low & Medium \\
8 & High & Medium & High \\
9 & High & High & High \\
\hline
\end{tabular}

\section{Performance Evaluation}

In order to assess the performance of the proposed approach, several tests have been made using the Network Simulator Version-2 (NS-2) [33] for the evaluation of the proposed HEM scheme and for simulating the BLE-based WN infrastructure. Simulations have been performed making a comparison with an approach without energy management and with the approaches proposed by Erol-Kantarci et al. [27] and Mahmood et al. [28] in which an HEM scheme, based on a WN, for a smart home is introduced. The main differences between the approaches presented in [27] and [28] and the solution presented in this work are that the algorithm introduced both by Erol-Kantarci et al. and Mahmood et al. does not take into account the feedback of the consumers, although it is similar to the one presented in this paper, and their considered WN is based on the IEEE 802.15.4 wireless protocol.

In this paper, the simulations have been carried out in the same scenario considered by Erol-Kantarci et al. and Mahmood et al., in order to have a direct comparison with their HEM scheme. Specifically, the scenario is composed of four loads, represented by a washer, a dryer, a dishwasher and a coffeemaker. The duration and the energy consumption of these appliances are vendor specific; however, the reference values for average load per cycle given in [34] have been used. The washer, the dryer, the dishwasher and the coffeemaker are assumed to consume $0.89 \mathrm{kWh}, 2.46 \mathrm{kWh}, 1.19 \mathrm{kWh}$ and $0.4 \mathrm{kWh}$, respectively, while the durations of their cycles are 30, 60, 90 and $10 \mathrm{~min}$. Moreover, an extra load has been supposed whose electricity consumption value varies randomly between $0 \mathrm{kWh}$ and $4 \mathrm{kWh}$. Therefore, $80 \%$ of the load is miscellaneous, and the remaining $20 \%$ is related to standby appliances. It is useful to note that peak hours can change both with days and seasons. For this reason, in this paper, the peak hours fall from 8 a.m.-2 p.m., as in the scenario considered by Erol-Kantarci et al. and Mahmood et al. As stated in [35], the electricity consumption behavior can be modeled as a Poisson random distribution that is a discrete random distribution. For this reason, even in this paper, the switching on of an appliance has been considered as a Poisson distribution. The switch on requests of appliances have been generated randomly, while the threshold value of power $\left(P_{t h r}\right)$, used in the proposed algorithm, has been set to $1 \mathrm{kWh}$. The simulations' duration has been between 10 days and 210 days (approximately seven months). The first 10 days are spared for warm up. The threshold 
value of delay $\left(D_{t h r}\right)$ has been set to $24 \mathrm{~h}$. Regarding the $\mathrm{WN}$, the traffic model, as mentioned above, has been considered as Poisson, and the NS-2 radio propagation model has been chosen as shadowing. On the contrary, as in the simulations of Erol-Kantarci et al. and Mahmood et al., the channel bit error rate has not been taken into account.

Figure 7 shows the electricity consumption pattern measured in a single day. It is clear that the proposed solution and the approaches of Erol-Kantarci et al. and Mahmood et al. shift loads from peak hours to off-peak hours efficiently. It is useful to remark that the percentage of load in peak hours is a ratio between the amount of load in peak hours to the total load. A high value of this ratio results in high electricity charges due to pricing tariffs. In peak hours, in order to provide monetary benefits to the consumer, the approaches reduce the percentage of the load. However, it is clear that the proposed solution obtains the best performance. This may be explained considering that the algorithm proposed by Erol-Kantarci et al. is quite different compared to the one introduced in this paper; on the contrary, in the algorithm proposed by Mahmood et al., different threshold values for $P_{t h r}$ and $D_{t h r}$ are used, and as a consequence, the performances are different from those obtained with the algorithm proposed in this work.

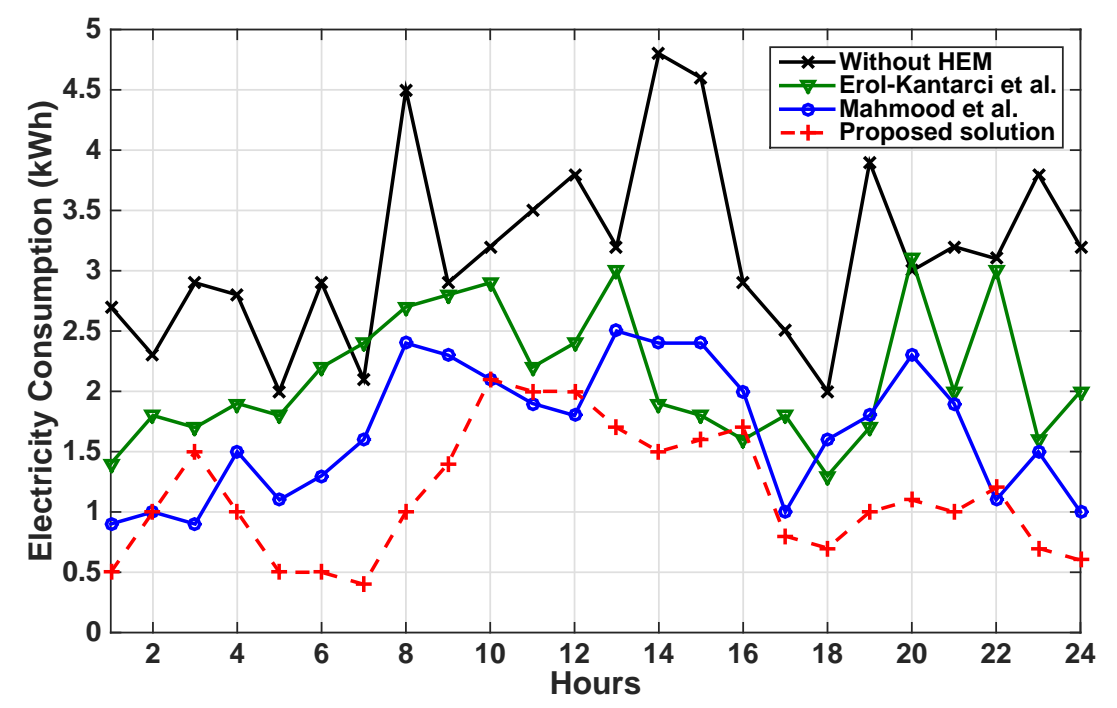

Figure 7. Peak load reduction comparison.

The contribution of the appliances to the average peak load is depicted in Figure 8. As it is possible to see, without an energy management approach, almost 0.3 of the load generated by the appliances takes place during peak periods. On the contrary, Mahmood et al.'s approach shifts those loads from peak times, and almost 0.12 of the total load is left in the peak hours, while Erol-Kantarci et al.'s approach obtains a value of about 0.13 . On the contrary, using the proposed solution, the peak load is reduced to 0.05. Even in this case, the best performances are those obtained with the proposed HEM solution. 


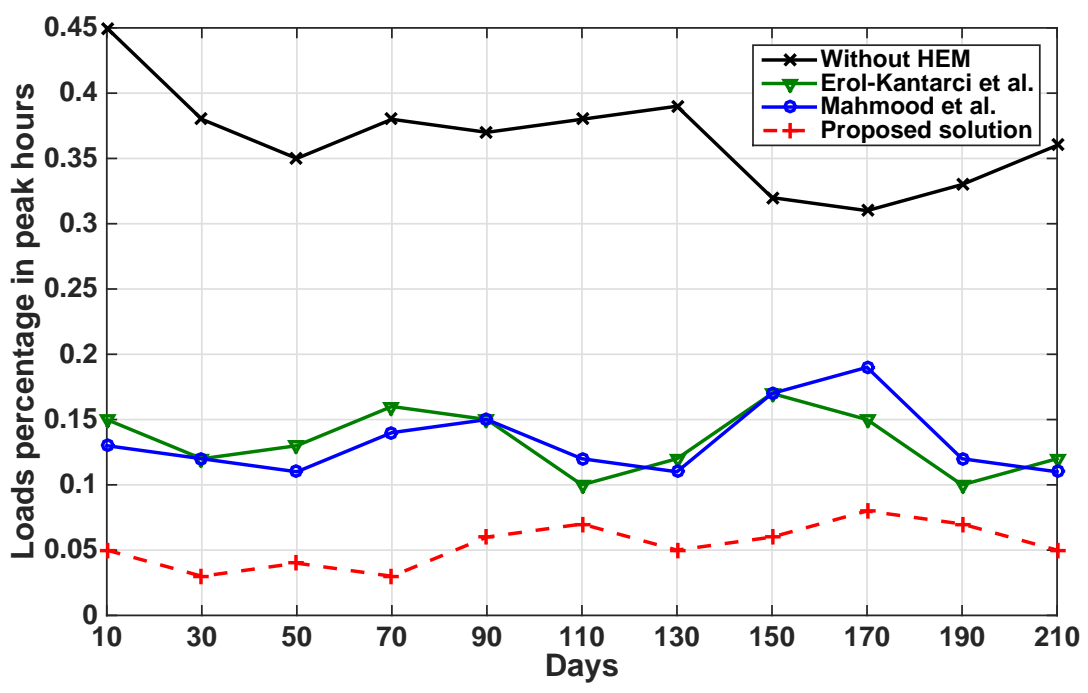

Figure 8. Load of the appliances ratio during peak hours.

The average delay experienced by the consumer is shown in Figure 9. It is necessary to note that without an energy management scheme, an appliance starts as soon as the consumer turns it on, and as a consequence, the delay is almost zero. For this reason, this case has not been included in the plot. For Mahmood et al.'s scheme, the delay fluctuates from 3-4 h, while using Erol-Kantarci et al.'s approach, the delay is almost equal, since it fluctuates from 3-4.5 h. On the contrary, in using the proposed solution, the delay is less than $3 \mathrm{~h}$ in most of the simulation. In fact, in the HEM algorithm introduced in this work, the consumer's feedback is taken into account in the FLC in order to improve the calculation of the delay. As a consequence, the delay can be updated if the consumer has already given one or more feedbacks in the past on the same appliance. The consumer's feedback represents clear indicators of his or her desired comfort level. It is useful to highlight that the delays shown in Figure 9 can be tolerable for several appliances, such as washers or dishwashers. On the contrary, for other appliances, such as coffeemakers, the consumer wants to start them immediately. For this reason, in the proposed approach, the consumer may deny or accept the schedule proposed by the EMU.

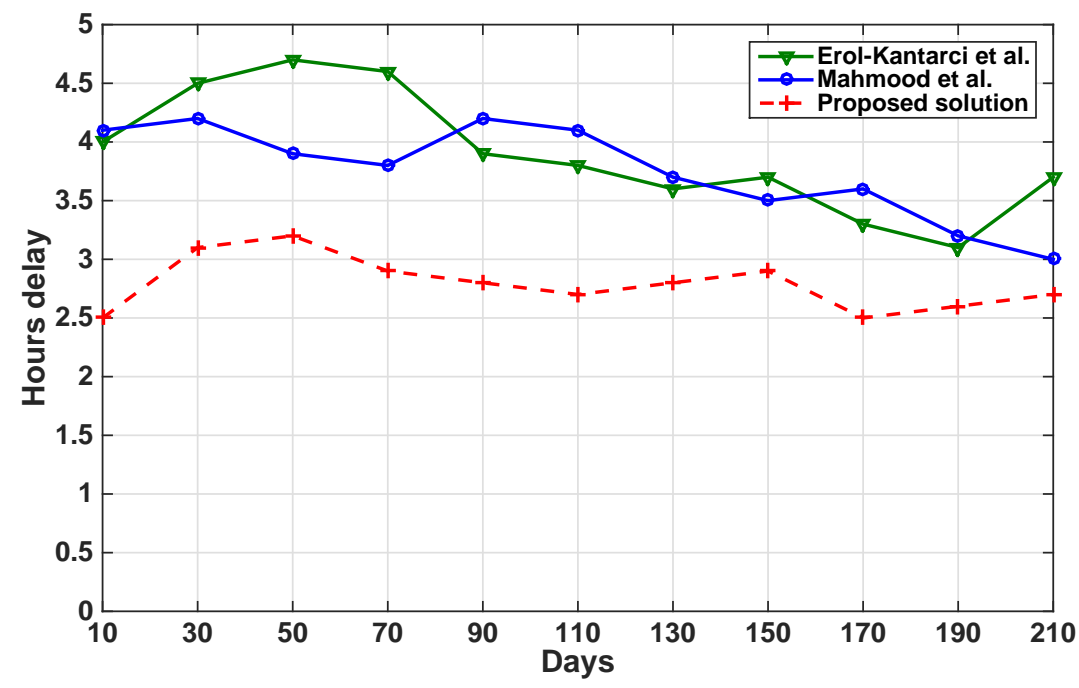

Figure 9. Average delay experienced by the consumer in HEM schemes. 
After evaluating the performance of the HEM solution proposed in this paper, it is useful to analyze what could be the economic benefits in its application. Regarding the monetary cost reduction, it is necessary to note that across the world, the electricity distributors have implemented various tariff schemes for peak load management. In this paper, the proposed scheme is based on a time-of-use tariff, and as a consequence, tariff pricing rates are different for different hours. In detail, the considered tariff is the one proposed by the Italian Enel [36], a multinational manufacturer and distributor of electricity and gas, whose costs are $0.0365 € / \mathrm{kWh}$ in off peak hours and $0.1725 € / \mathrm{kWh}$ in peak hours (from 8 a.m.-7 p.m.). The electricity consumption cost of a single home has been simulated both when no energy management is applied and when the approaches of Erol-Kantarci et al. and Mahmood et al. and the proposed solution are applied. The simulation results for a seven month electricity bill is shown in Figure 10. It is useful to note that the electricity bill increases with increasing months, because the bill is calculated cumulatively. Even in this case, the best results are obtained with the solution proposed in this paper that allows one to achieve a higher monetary cost reduction.

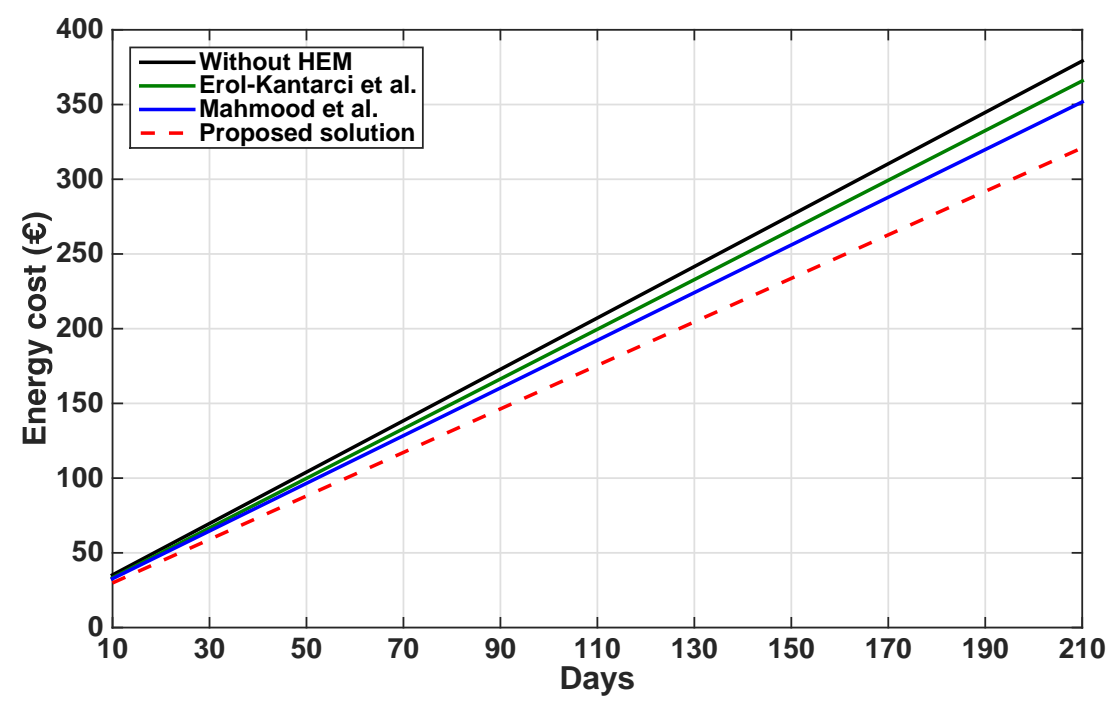

Figure 10. The electricity consumption cost.

The performance of the proposed $\mathrm{WN}$, in terms of the packet delivery ratio, end-to-end delay and jitter, has been evaluated in the simulations. It is useful to remind the reader that the delivery ratio is the ratio of the number of successfully-received packets to the number of sent packets. The end-to-end delay represents the interval between sending a packet from the application layer of the source and receiving it at the application layer of the destination. The jitter is the difference between the delays experienced by the packets. Even in this case, the performance has been compared with those obtained by the WN of Erol-Kantarci et al. and Mahmood et al. However, it is necessary to remind the reader that their WN is based on IEEE 802.15.4, and they have shown the impact of the variation in the packet size. In fact, they have varied the packet sizes from 32 bytes-128 bytes. For this reason, the packet size that obtains the best result in their approaches has been considered for the comparison. On the contrary, in this work, the WN is based on BLE 4.0, which supports only very short data packets, whose maximum size is 27 bytes. For this reason, the packet size has not been varied. 
As depicted in Figure 11, considering the results obtained by Erol-Kantarci et al., the best results, about $77 \%$, are obtained with a packet size of 32 bytes. This same packet size gets the best results in the approach of Mahmood et al., about $80 \%$. On the contrary, the packet delivery ratio of the BLE-based WN proposed in this work is almost $90 \%$. In fact, BLE data packets are more efficient when sending small amounts of data infrequently.

The end-to-end delay is shown in Figure 12. In the IEEE 802.15.4-based WN of Erol-Kantarci et al., it is around $0.65 \mathrm{~s}$, with a packet size of 96 bytes, while using the approach of Mahmood et al., the delay is around $0.77 \mathrm{~s}$ with a packet size of 128 bytes. In BLE, it is possible to have a connection setup and data transfer as low as $3 \mathrm{~ms}$. However, in the worst case, the delay can be also higher than $1.28 \mathrm{~s}$. In order to make a direct comparison among the approaches with the same packet size, Figure 12 also shows the results obtained applying the approaches of Erol-Kantarci et al. and Mahmood et al. using the BLE protocol, whose end-to-end delay is around $0.33 \mathrm{~s}$ and $0.27 \mathrm{~s}$, respectively. These values are lower than those obtained using the IEEE 802.15.4 protocol. On the contrary, the end-to-end delay measured in the proposed BLE-based WN is around $0.17 \mathrm{~s}$, a value lower than that obtained in the best case by Erol-Kantarci et al. and Mahmood et al. This highlights the goodness of BLE, which allows an application to form a connection and then to transfer authenticated data in a few seconds for a short communication burst before quickly tearing down the connection.

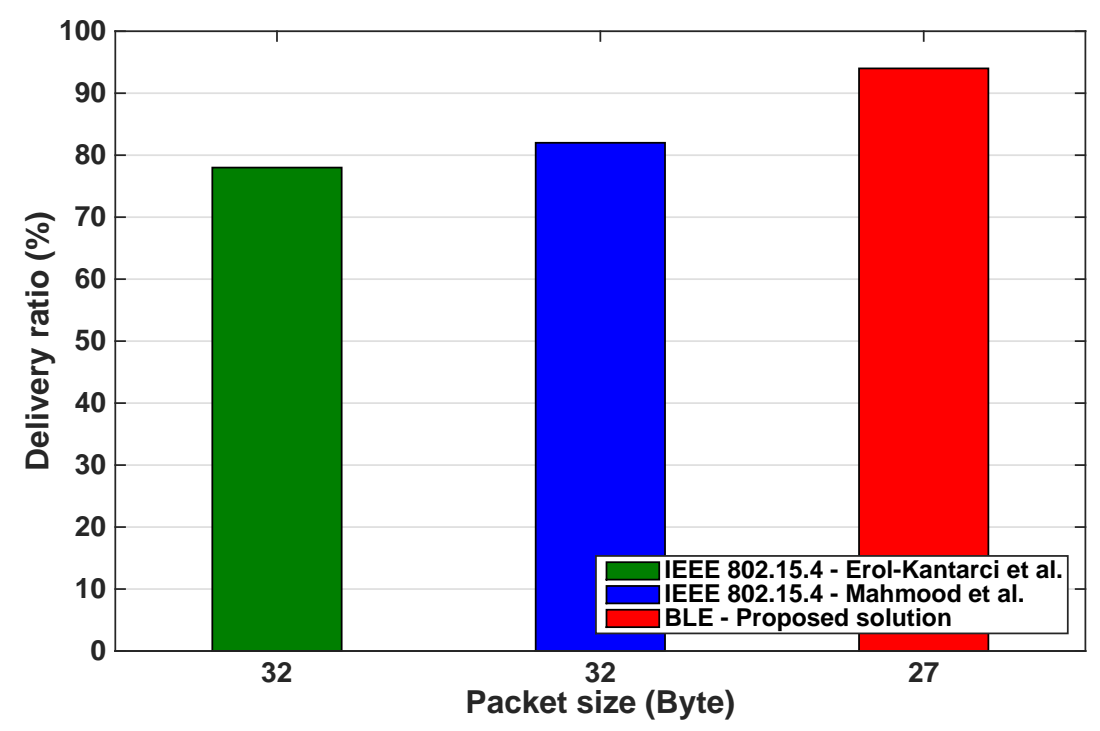

Figure 11. Delivery ratio of the wireless networks (WNs). 


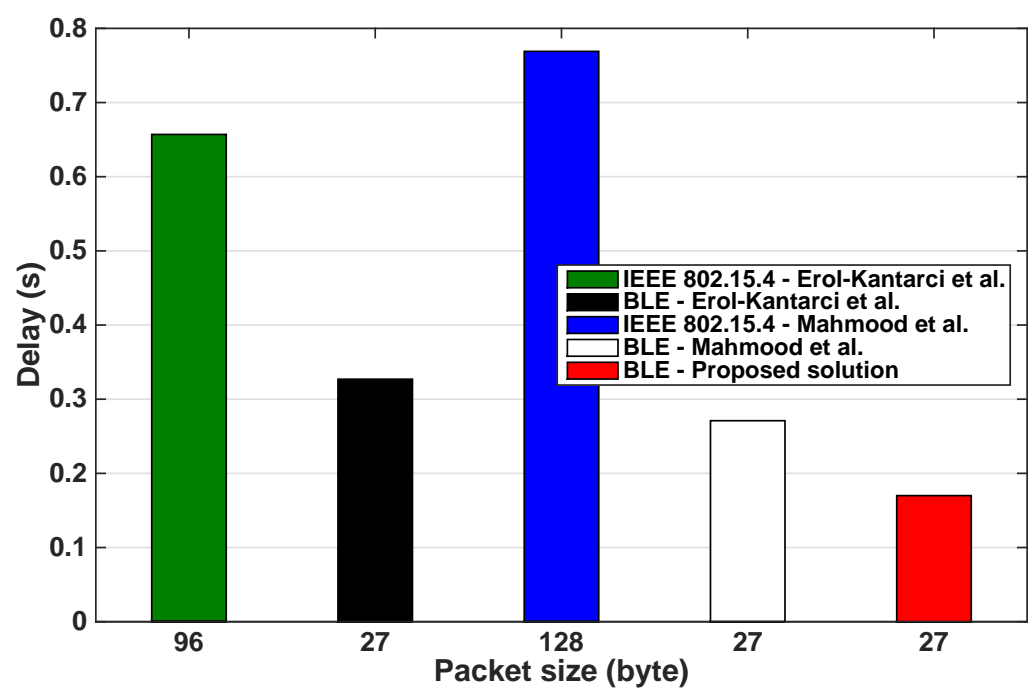

Figure 12. End-to-end delay of the WNs.

The jitter is another important parameter, because high jitter values mean that consumers may experience variable end-to-end delays when they are communicating with the EMU. The jitter values are shown in Figure 13. In all of the approaches, the values are low, less than $0.05 \mathrm{~s}$, which is negligible considering the human response times. However, even in this case, the proposed WN obtains a better result than the ones of Erol-Kantarci et al. and Mahmood et al.

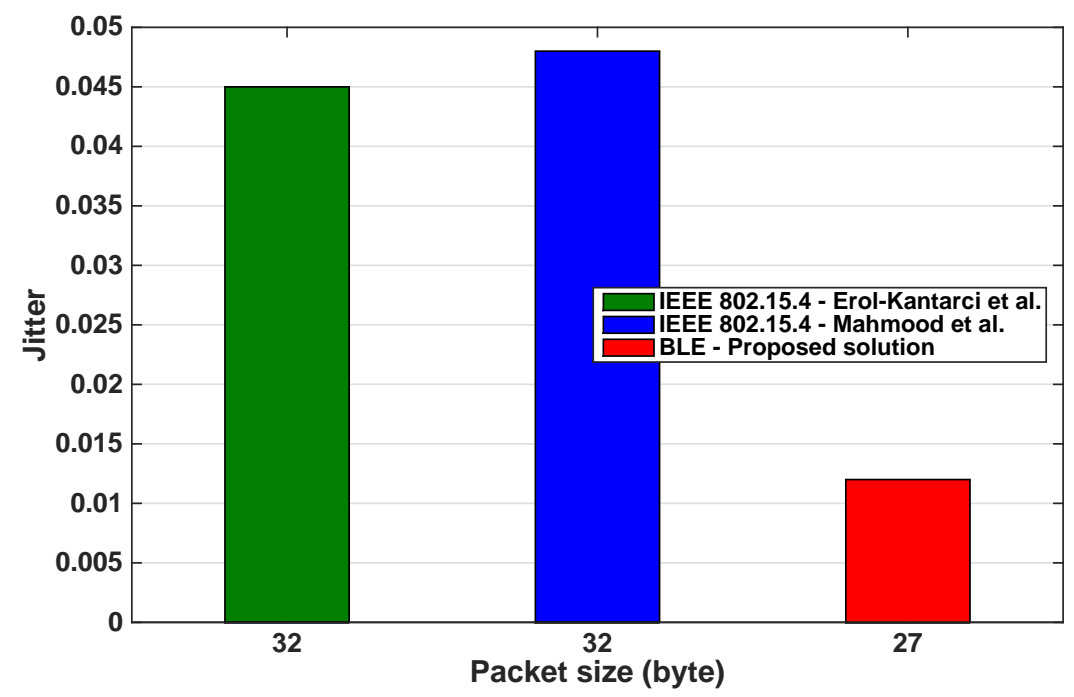

Figure 13. Jitter of the WNs.

\section{Conclusions}

An energy management approach for smart homes that combines a Wireless Network based on Bluetooth Low Energy, for the communication among home appliances, with a Home Energy Management scheme, improved by a consumer fuzzy-based approach, has been proposed in this paper. This combination has been implemented because residential energy management, smart appliances, WNs, and their integration into smart home network applications are becoming popular topics. 
Furthermore, a HEM implementation can lead to socially and economically beneficial environment by addressing consumers' and utilities concerns.

The main issue of this work is the reduction, guaranting a consumer comfort, the energy consumption and limiting the impact of standby appliances. It was been carried out thanks to a synergy between a BLE-based WN and a fuzzy-based algorithm for HEM.

Several simulations have been carried out in order to evaluate the electricity consumption pattern measured in a single day, the contribution of the appliances on the average peak load and the average delay experienced by the consumer, and the benefits of fuzzy solution that search the best tradeoff between consumer will and energy consumption. Simulation results have clearly shown that the proposed solution responds to the challenge of new research issues, that are studing methods and approaches based on online user feedbacks.

Our proposed approach is quite efficient in terms of contribution to comfort level of the consumer by putting a limit on the delay factor of an appliance, as well as reducing the peak load demand and electricity consumption charges and allows to achieve a concrete monetary cost reduction.Summarizing in case of the appliance cycle is shifted to off peak hours and the delay goes beyond the limit the cycle is retained and the appliance is switched on immediately. In addition, in order to have a proper delay value, the consumers feedback provided in the past on the appliances is taken into account and is used as an input parameter of a FLC. As a result, the value of the delay can be increased or decreased, always taking into account the consumer's feedback that represent clear indicators on its desired comfort level. Finally, the performance of the WN has been validated and the results highlight the goodness of the BLE network in terms of packet delivery ratio, delay, and jitter compared to other literature works, that implement IEEE 802.15.4 technology.

\section{Author Contributions}

All of the authors have contributed toward developing the ideas. All of the authors have collaborated to obtain the results, the performance evaluation and in the definition of reference scenarios. All of the authors have been involved in preparing the manuscript.

\section{Conflicts of Interest}

The authors declare no conflict of interest.

\section{References}

1. Liu, Y. Study on smart home system based on internet of things technology. In Informatics and Management Science IV; Du, W., Ed.; Springer: London, UK, 2013; Volume 207, pp. 73-81.

2. Yohanis, Y.G.; Mondol, J.D.; Wright, A.; Norton, B. Real-life energy use in the UK: How occupancy and dwelling characteristics affect domestic electricity use. Energy Build. 2008, 40, 1053-1059.

3. Barbato, A.; Capone, A. Optimization models and methods for demand-side management of residential users: A survey. Energies 2014, 7, 5787-5824.

4. Torriti, J. A review of time use models of residential electricity demand. Renew. Sustain. Energy Rev. 2014, 37, 265-272. 
5. Mendes, T.D.P.; Godina, R.; Rodrigues, E.M.G.; Matias, J.C.O.; Catalao, J.P.S. Smart home communication technologies and applications: Wireless protocol assessment for home area network resources. Energies 2015, 8, 7279.

6. Ahmed, M.A.; Kang, Y.C.; Kim, Y.C. Communication network architectures for smart-house with renewable energy resources. Energies 2015, 8, 8716.

7. Collotta, M.; Messineo, A.; Nicolosi, G.; Pau, G. A dynamic fuzzy controller to meet thermal comfort by using neural network forecasted parameters as the input. Energies 2014, 8, 4727.

8. Bluetooth Specification Version 4.0, Bluetooh SIG; 2010. Available online: https://www.bluetooth.org/en-us/specification/adopted-specifications (accessed on 20 August 2015).

9. IEEE. IEEE Standard for Information Technology-Telecommunications and Information Exchange between Systems-Local and Metropolitan Area Networks- Specific Requirements Part 15.4: Wireless Medium Access Control (MAC) and Physical Layer (PHY) Specifications for Low-Rate Wireless Personal Area Networks (WPANs); Technical Report; IEEE: New York, NY, USA, 2006.

10. IEEE. IEEE Std 802.11-2007 for Information Technology-Telecommunications and Information Exchange between Systems-Local and Metropolitan Area Networks-Specific Requirements-Part 11: Wireless LAN Medium Access Control (MAC) and Physical Layer (PHY) Specifications, C1-1184; IEEE: New York, NY, USA, 2007.

11. Serna, M.; Sreenan, C.; Fedor, S. A visual programming framework for wireless sensor networks in smart home applications. In Proceedings of the 2015 IEEE Tenth International Conference on Intelligent Sensors, Sensor Networks and Information Processing (ISSNIP), Singapore, Singapore, 7-9 April 2015; pp. 1-6.

12. Gabriele, T.; Pantoli, L.; Stornelli, V.; Chiulli, D.; Muttillo, M. Smart power management system for home appliances and wellness based on wireless sensors network and mobile technology. In Proceedings of the Associazione Italiana Sensori e Microsistemi Annual Conference (2015 XVIII AISEM), Trento, Italy, 3-5 February 2015; pp. 1-4.

13. Keshtkar, A.; Arzanpour, S.; Keshtkar, F.; Ahmadi, P. Smart residential load reduction via fuzzy logic, wireless sensors, and smart grid incentives. Energy Build. 2015, 104, 165-180.

14. IEEE. IEEE Approved Draft Standard for Low-Frequency (less than $500 \mathrm{kHz}$ ) Narrowband Power Line Communications for Smart Grid Applications-Amendment 1; IEEE P1901.2a/D0.3; IEEE: New York, NY, USA, 2015; pp. 1-32.

15. Li, M.; Lin, H.J. Design and implementation of smart home control systems based on wireless sensor networks and power line communications. IEEE Trans. Ind. Electron. 2015, 62, 4430-4442.

16. Han, J.; sic Choi, C.; Park, W.K.; Lee, I.; Kim, S.H. Smart home energy management system including renewable energy based on ZigBee and PLC. IEEE Trans. Consum. Electron. 2014, 60, 198-202.

17. Pasdar, A.; Cavdar, I.; Sozer, Y. Power-line impedance estimation at FCC band based on intelligent home appliances status detection algorithm through their individual energy and impedance signatures. IEEE Trans. Power Deliv. 2014, 29, 1407-1416.

18. Freescale. KW4x: Kinetis KW40Z/30Z/20Z - Bluetooth Low Energy, 802.15.4 Wireless Microcontrollers (MCUs). Available online: http://www.freescale.com (accessed on 25 September 2015).

19. CSR. CSR101x Product Family. Available online: http://www.csr.com/products/bluetooth-smart-csr 101x-product-family (accessed on 25 September 2015). 
20. dialog SEMICONDUCTOR. SmartBond ${ }^{T M}$ DA1458x Product Family. Available online: http://www.dialog-semiconductor.com/sites/default/files/da14580-family-product-brief.pdf (accessed on 25 September 2015).

21. Bluetooth Smart SoCs. Available online: http://www.nxp.com (accessed on 25 September 2015).

22. Broadcom. WICED. Available online: http://www.broadcom.com (accessed on 25 September 2015).

23. Gungor, V.; Lu, B.; Hancke, G. Opportunities and challenges of wireless sensor networks in smart grid. IEEE Trans. Ind. Electron. 2010, 57, 3557-3564.

24. Jang, W.; Healy, W. Wireless sensor network performance metrics for building applications. Energy Build. 2010, 42, 862-868.

25. Ulukus, S.; Yener, A.; Erkip, E.; Simeone, O.; Zorzi, M.; Grover, P.; Huang, K. Energy harvesting wireless communications: A review of recent advances. IEEE J. Sel. Areas Commun. 2015, PP, 1-22.

26. Mohsenian-Rad, A.H.; Leon-Garcia, A. Optimal residential load control with price prediction in real-time electricity pricing environments. IEEE Trans. Smart Grid 2010, 1, 120-133.

27. Erol-Kantarci, M.; Mouftah, H. Wireless sensor networks for cost-efficient residential energy management in the smart grid. IEEE Trans. Smart Grid 2011, 2, 314-325.

28. Mahmood, A.; Khan, I.; Razzaq, S.; Najam, Z.; Khan, N.; Rehman, M.; Javaid, N. Home appliances coordination scheme for energy management (HACS4EM) using wireless sensor networks in smart grids. Procedia Comput. Sci. 2014, 32, $469-476$.

29. Tompros, S.; Mouratidis, N.; Draaijer, M.; Foglar, A.; Hrasnica, H. Enabling applicability of energy saving applications on the appliances of the home environment. IEEE Netw. 2009, 23, 8-16.

30. Zadeh, L.A. The concept of a linguistic variable and its application to approximate reasoning-II. Inf. Sci. 1975, 301-357.

31. Pedrycz, W. Why triangular membership functions? Fuzzy Sets Syst. 1994, 64, 21-30.

32. Runkler, T.A.; Glesner, M. DECADE-Fast centroid approximation defuzzification for real time fuzzy control applications. In Proceedings of the Association for Computing Machinery Symposium on Applied Computing (SAC '94), New York, NY, USA, 1994; pp. 161-165.

33. Breslau, L.; Estrin, D.; Fall, K.; Floyd, S.; Heidemann, J.; Helmy, A.; Huang, P.; McCanne, S.; Varadhan, K.; Xu, Y.; et al. Advances in network simulation. Computer 2000, 33, 59-67.

34. Stamminger, R. Synergy potential of smart appliances. In A Report Prepared as Part of the EIE Project-Smart Domestic Appliances in Sustainable Energy Systems (Smart-A); Bonn University: Bonn, Germany, 2008.

35. Lai, S.; Messier, G.; Zareipour, H.; Wai, C. Wireless network performance for residential demand-side participation. In Proceedings of the 2010 IEEE PES Innovative Smart Grid Technologies Conference Europe (ISGT Europe), Gothenburg, Sweden, 11-13 October 2010; pp. 1-4.

36. ENEL. Energia Pura Bioraria. Available online: https://www.enelenergia.it (accessed on 20 August 2015).

(c) 2015 by the authors; licensee MDPI, Basel, Switzerland. This article is an open access article distributed under the terms and conditions of the Creative Commons Attribution license (http://creativecommons.org/licenses/by/4.0/). 